\title{
Waugh Type Enneamolybdomanganate(IV) Catalyzed Synthesis of Polyhydrquinoline Through Hantzsch Multi-Component Condensation
}

\author{
Amit R. Supale and Gavisiddappa S. Gokavi* \\ Kinetics and Catalysis Laboratory, Department of Chemistry, Shivaji University, Kolhapur-416004, India
}

\begin{abstract}
An efficient one pot coupling of aldehydes, dimedone, ammonium acetate and ethyl acetoacetate by using catalytic amount of enneamolybdomanganate(IV) is reported. Various polyhydroquinoline derivatives have been prepared in high yields and comparatively less reaction time.
\end{abstract}

Keywords: Waugh type enneamolybdomanganate(IV), Polyhydroquinoline derivatives, Hantzsch reaction, Multicomponent reaction.

\section{INTRODUCTION}

In recent decades, multicomponent reactions (MCR's) have gain wide applicability in the field of synthetic organic chemistry as they increase the efficiency of the reaction and decrease the number of laboratory operations along with quantities of solvent and chemicals used. These methods also considerably reduce the reaction time and facilitate the yield of products than the normal multiple step methods. Therefore, due to increased environmental responsibility and develop more efficient catalytic systems in synthetic organic chemistry attempts have always been made by the scientists to improve the known protocols. Such developments leads to prevail over one or more difficulties encountered in already reported systems. The synthesis of 1,4-dihydropyridyl compounds $(1,4-\mathrm{DHPs})$ is of great importance in chemistry due to its pharmacological and biological activities [1]. The dihydropyridyl compounds are calcium channel blockers hence suitable for treatment of cardiovascular diseases [2]. 1,4dihydropyridine compounds possess a variety of biological activities such as vasodilators, antiatherosclerotic, antitumor, geroprotective, antidiabetic activities [3]. These also act as chemosensitizer in tumor therapy and are neuroprotectantcerebral antischemic agent [4]. The wide utility of 1, 4-DHPs is the main reason for its synthesis by different methods. Several methods have been reported for the synthesis of polyhydroquinoline derivatives. The classical method [5] involves the three component condensation of aldehydes with ethyl acetoacetate and ammonia in acetic acid or refluxing ethanol. But this method suffers from drawbacks such as low yield. Hence attempts have been made to find out various alternatives for synthesis of these compounds [6]. Many of them involve harsh reaction condition, difficult workup procedure, generation of side products and long reaction time. The increasing demand of newer methods having suitability generating high yields and prevails over previous method is of current interest in chemistry.

*Address correspondence to this author at the Kinetics and Catalysis Laboratory, Department of Chemistry, Shivaji University, Kolhapur-416004, India; Tel: (0231) 2690571; Fax: 91-0231-2692333;

E-mail: gsgokavi@hotmail.com
The properties of polyoxometalate (POM) anions make them suitable for application in almost all aspects of chemistry and material science [7]. Most of the reactions of POM anions are concentrated on the Keggin type POM containing hetero atom in its higher oxidation state. In comparison with Keggin and Dawson type of salts, less attention is given to other type of POM anions. The polyoxometalates like enneamolybdomanganate (IV) is previously used [8] as oxidant in electron transfer reactions. In continuation of our efforts in various organic transformations [9] we report herein the catalytic activitiy of Waugh type polyoxometalate for four component condensation to synthesize 1,4-DHPs. The condensation is carried out conventionally at room temperature in ethanol as shown in scheme $\mathbf{1 .}$

\section{EXPERIMENTAL SECTION}

All the products have been reported previously and were characterized by comparison of their physical and spectral data. ${ }^{1} \mathrm{H}$ NMR, ${ }^{13} \mathrm{C}$ NMR spectra were recorded on Bruker Avance $300 \mathrm{MHz}$ spectrometer. Mass spectra were recorded on Shimadzu GCMS-QP2010. Melting points were determined on an open capillary and are uncorrected. IR was recorded on Perkin Elmer-spectra one. Aldehydes, Ethanol, Dimedone, ethyl acetoacetate, ammonium acetate were purchased from SD Fine chemicals (Mumbai, India). The solvents used such as ethanol, ethyl acetate are of AR grade having high purity $(99 \%)$. The dimedone is of AR grade $(99 \%)$. The aldehydes, ethyl acetoacetate and ammonium acetate are highly pure $(>98 \%)$ and used without further purification. All yields refer to isolated yields.

\subsection{Preparation of Catalyst}

The ammonium salt of $\mathrm{Mn}^{\mathrm{IV}}$ complex, $\left(\mathrm{NH}_{4}\right)_{6}\left[\mathrm{Mn}^{\mathrm{IV}}\right.$ $\mathrm{Mo}_{9} \mathrm{O}_{32}$ ] was prepared by reported method [10] as follows; $50 \mathrm{gm}$ of ammonium molybdate was dissolved in $200 \mathrm{ml}$ of water and excess of hydrogen peroxide as oxidant was added to it and the resultant solution was heated to $95^{\circ} \mathrm{C}$. To this hot solution, $5 \mathrm{gm}$ of $\mathrm{MnSO}_{4} \cdot \mathrm{H}_{2} \mathrm{O}$ in $50 \mathrm{ml}$ of water was added slowly with constant stirring. The resultant orange-red colored solution was boiled for 10 minutes and quickly filtered and cooled. The orange-red colored crystals were recrystalized thrice from hot $\left(70^{\circ} \mathrm{C}\right)$ water. 


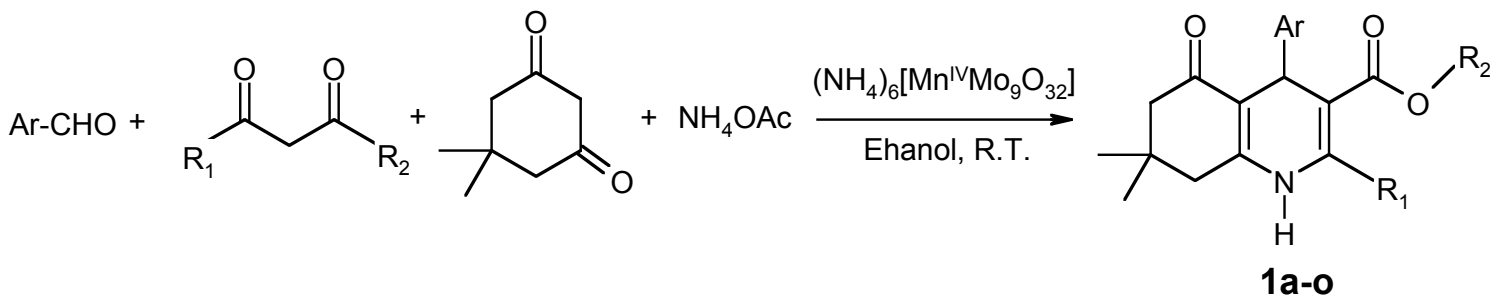

Scheme 1. Synthesis of polyhydroquinoline derivatives.

\subsection{Catalyst Characterization}

The complex $\left(\mathrm{NH}_{4}\right)_{6}\left[\mathrm{Mn}^{\mathrm{IV}} \mathrm{Mo}_{9} \mathrm{O}_{32}\right] \cdot 3 \mathrm{H}_{2} \mathrm{O}$, was analyzed by AAS. The solution for AAS analysis was prepared by dissolving $100 \mathrm{mg}$ of recrystalized sample in double distilled water and $5 \mathrm{ml}$ of this stock solution was diluted to $100 \mathrm{ml}$. The diluted solution was used for AAS analysis of $\mathrm{Mn}$ and Mo using Perkin-Elmer AAnalyst-300 instrument. The complex $\left(\mathrm{NH}_{4}\right)_{6}\left[\mathrm{Mn}^{\mathrm{IV}} \mathrm{Mo}_{9} \mathrm{O}_{32}\right] \cdot 3 \mathrm{H}_{2} \mathrm{O}$ shows (Theoretical): Mn$3.501 \%$ (3.4493\%) and Mo- $54.217 \%$ (54.2125\%).

The complex $\left(\mathrm{NH}_{4}\right)_{6}\left[\mathrm{Mn}^{\mathrm{IV}} \mathrm{Mo}_{9} \mathrm{O}_{32}\right]$ contains $\mathrm{Mn}^{\mathrm{IV}}$ as a hetero-atom surrounded by octahedral groups of $\mathrm{MoO}_{6}{ }^{2-}$. In the IR-spectrum of $\mathrm{Mn}^{\text {IV }}$ complex, the peaks obtained at $3521 \mathrm{~cm}^{-1}, 933 \mathrm{~cm}^{-1}, 918 \mathrm{~cm}^{-1}$ and $900 \mathrm{~cm}^{-1}$ corresponds to lattice water molecules, Mo-O stretching, $\mathrm{Mn}^{\mathrm{IV}}-\mathrm{O}$ stretching and tetrahedral $\mathrm{MoO}_{4}{ }^{2-}$ groups, respectively [11].

\subsection{General Procedure for Synthesis of Hantzsch Poly- hydroquinoline Derivatives}

In a typical experimental procedure aldehydes (1 $\mathrm{mmol})$, ethyl acetoacetate $(1 \mathrm{mmol})$, dimedone $(1 \mathrm{mmol})$, ammonium acetate $(1 \mathrm{mmol})$ and catalyst $\left(\mathrm{NH}_{4}\right)_{6}\left[\mathrm{Mn}^{\mathrm{IV}} \mathrm{Mo}_{9} \mathrm{O}_{32}\right](2$ mol\%) were taken in $25 \mathrm{ml}$ round bottom flask. $2.5 \mathrm{ml}$ ethanol was added to it. The reaction mixture was stirred at room temperature. The progress of the reaction was monitored by TLC. After completion of the reaction the mixture was extracted with ethyl acetate. Organic layer was dried on anhydrous sodium sulphate. Solvent was evaporated to get required product. Further aqueous layer was concentrated to get catalyst back and it was recycled three times.

\section{RESULTS AND DISCUSSION}

Enneamolybdomanganate(IV) is one of the stable heteropolymolybdate containing $\mathrm{Mn}^{\mathrm{IV}}$ as a hetero atom and is noncentrosymmetric. To study the catalytic activity of $\left(\mathrm{NH}_{4}\right)_{6}\left[\mathrm{Mn}^{\mathrm{IV}} \mathrm{Mo}_{9} \mathrm{O}_{32}\right]$, benzaldehyde was chosen as a model substrate and reaction was carried out by simply mixing it with dimedone, ethyl acetoacetate and ammonium acetate in ethanol at room temperature. The corresponding polyhydroquinoline derivative was obtained in high yield (90\%).

The $2 \mathrm{~mol} \%$ of catalyst Enneamolybdomanganate(IV) is sufficient to carry out the condensation. Increasing amount of catalyst did not lead to considerable improvement in the yield of polyhydroquinoline derivatives. The results are summarized in Table $\mathbf{1}$. To express the generality of reaction various aromatic aldehydes were tested along with $\beta$-keto esters (ethyl acetoacetate, methyl acetoacetete) for this reaction. Aldehydes containing electron donating substituents such as 4-OMe benzaldehyde (giving 1e, 88\%) and electron withdrawing substituents such as $3-\mathrm{NO}_{2}$ (giving 1b, 86\%) underwent the conversion smoothly. Under this procedure vinyl aldehydes (giving 1g, 85\%; 10, 84\%) heterocyclic aldehydes like furaldehyde (giving $\mathbf{1 h}, 81 \%$ ) were also worked well. Aldehydes having various functional groups were tolerated during reaction giving high yields of respected polyhydroquinoline derivatives (81-92\%). The results are summarized in Table 2.

Table 1. Effect of Catalyst Concentration

\begin{tabular}{|c|c|c|c|}
\hline Sr. No. & Catalyst (mol\%) & Time (h) & Yield (\%) $^{\mathbf{b}}$ \\
\hline \hline 1 & 1 & 2 & 70 \\
\hline 2 & 2 & 1.5 & 90 \\
\hline 3 & 5 & 1.5 & 91 \\
\hline 4 & 10 & 1.5 & 91 \\
\hline
\end{tabular}

$\mathrm{Mn}(\mathrm{IV})$ act as a mild acid catalyst in this coupling reaction. Aqueous ammonium acetate is used in the reaction mixture and hence catalyst dissolved in it recovered from aqueous layer after the extraction procedure. This was recycled for three times successfully.

\section{Physical and Spectra Data for Selected Compounds:}

2,7,7-Trimethyl-5-Oxo-4-Phenyl-1,4,5,6,7,8-Hexahydroquinoline-3-Carboxylic Acid Ethyl Ester (1a)

Mp: $203-205{ }^{0} \mathrm{C}$.

IR (KBr): 3289, 3080, 2959, 1698, $1610 \mathrm{~cm}^{-1}$.

${ }^{1} \mathrm{H} \mathrm{NMR}\left(\mathrm{CDCl}_{3}, 300 \mathrm{MHz}\right): \mathrm{d} 0.91(\mathrm{~s}, 3 \mathrm{H}), 1.05(\mathrm{~s}, 3 \mathrm{H})$, $1.17(\mathrm{t}, \mathrm{J} 1 / 47.1 \mathrm{~Hz}, 3 \mathrm{H}), 2.14-2.20(\mathrm{~m}, 4 \mathrm{H}), 2.28$ (s, 3H), 4.03 $\left(\mathrm{q}, \mathrm{J}^{1} / 47.1 \mathrm{~Hz}, 2 \mathrm{H}\right), 5.02(\mathrm{~s}, 1 \mathrm{H}), 5.96(\mathrm{~s}, 1 \mathrm{H}), 7.04-7.09(\mathrm{~m}$, 1H), 7.14-7.19 (m, 2H), 7.23-7.26 (m, 2H). ${ }^{13} \mathrm{C}$ NMR $\left(\mathrm{CDCl}_{3}, 300 \mathrm{MHz}\right)$ : d 14.18, 19.45, 27.16, 29.41, 32.72, $36.53,41.16,50.70,59.81,100.33,106.29,112.61,126$, $127.86,143.83,146.99,147,167.68,195.75$

MS (ESI) $\mathrm{m} / \mathrm{z}(\mathrm{M}+\mathrm{H})^{+}$Calculated for $\mathrm{C}_{21} \mathrm{H}_{26} \mathrm{NO}_{3}: 340.2$, Found: 340 .

2,7,7-Trimethyl-5-Oxo-4-(3-Nitrophenyl)-1,4,5,6,7,8-Hexahydroquinoline-3-Carboxylic Acid Ethyl Ester (1b)

Mp: $177-179{ }^{\circ} \mathrm{C}$.

${ }^{1} \mathrm{H}$ NMR $\left(\mathrm{CDCl}_{3}, 300 \mathrm{MHz}\right): \mathrm{d} 0.9$ (s, 3H), 1.06 (s, 3H), $1.17(\mathrm{t}, \mathrm{J} 1 / 47.1 \mathrm{~Hz}, 3 \mathrm{H}), 2.08-2.38(\mathrm{~m}, 7 \mathrm{H}), 4.03(\mathrm{q}, \mathrm{J} 1 / 47.1$ $\mathrm{Hz}, 2 \mathrm{H}), 5.12(\mathrm{~s}, 1 \mathrm{H}), 6.36(\mathrm{~s}, 1 \mathrm{H}), 7.23(\mathrm{~d}, 1 \mathrm{H}), 7.35(\mathrm{t}$, $\mathrm{J} 1 / 47.9 \mathrm{~Hz}, 1 \mathrm{H}), 7.68(\mathrm{~d}, \mathrm{~J} 1 / 47.9 \mathrm{~Hz}, 1 \mathrm{H}), 7.71(\mathrm{~m}, 1 \mathrm{H}), 7.93$ (m, 1H). 
Table 2. Synthesis of Polyhydroquinoline Derivatives ${ }^{a}$

\begin{tabular}{|c|c|c|c|c|c|c|}
\hline Entry & Ar & $\mathbf{R}_{1}$ & $\mathbf{R}_{2}$ & Product & Time (h) & Yield (\%) \\
\hline $1 \mathbf{a}$ & $\mathrm{C}_{6} \mathrm{H}_{5}$ & $\mathrm{CH}_{3}$ & $\mathrm{C}_{2} \mathrm{H}_{5}$ & & 1.5 & 90 \\
\hline 1b & $3-\mathrm{NO}_{2} \mathrm{C}_{6} \mathrm{H}_{4}$ & $\mathrm{CH}_{3}$ & $\mathrm{C}_{2} \mathrm{H}_{5}$ & & 1.5 & 86 \\
\hline $1 \mathrm{c}$ & $4-\mathrm{NO}_{2} \mathrm{C}_{6} \mathrm{H}_{4}$ & $\mathrm{CH}_{3}$ & $\mathrm{C}_{2} \mathrm{H}_{5}$ & & 1.5 & 88 \\
\hline 1d & $3-\mathrm{OMeC}_{6} \mathrm{H}_{4}$ & $\mathrm{CH}_{3}$ & $\mathrm{C}_{2} \mathrm{H}_{5}$ & & 1.5 & 85 \\
\hline $1 e$ & 4- $\mathrm{OMeC}_{6} \mathrm{H}_{4}$ & $\mathrm{CH}_{3}$ & $\mathrm{C}_{2} \mathrm{H}_{5}$ & & 2 & 88 \\
\hline 1f & $4-\mathrm{OHC}_{6} \mathrm{H}_{4}$ & $\mathrm{CH}_{3}$ & $\mathrm{C}_{2} \mathrm{H}_{5}$ & & 1.5 & 86 \\
\hline $1 \mathrm{~g}$ & $\mathrm{C}_{6} \mathrm{H}_{5} \mathrm{CH}=\mathrm{CH}$ & $\mathrm{CH}_{3}$ & $\mathrm{C}_{2} \mathrm{H}_{5}$ & & 2 & 85 \\
\hline
\end{tabular}


(Table 2) contd.....

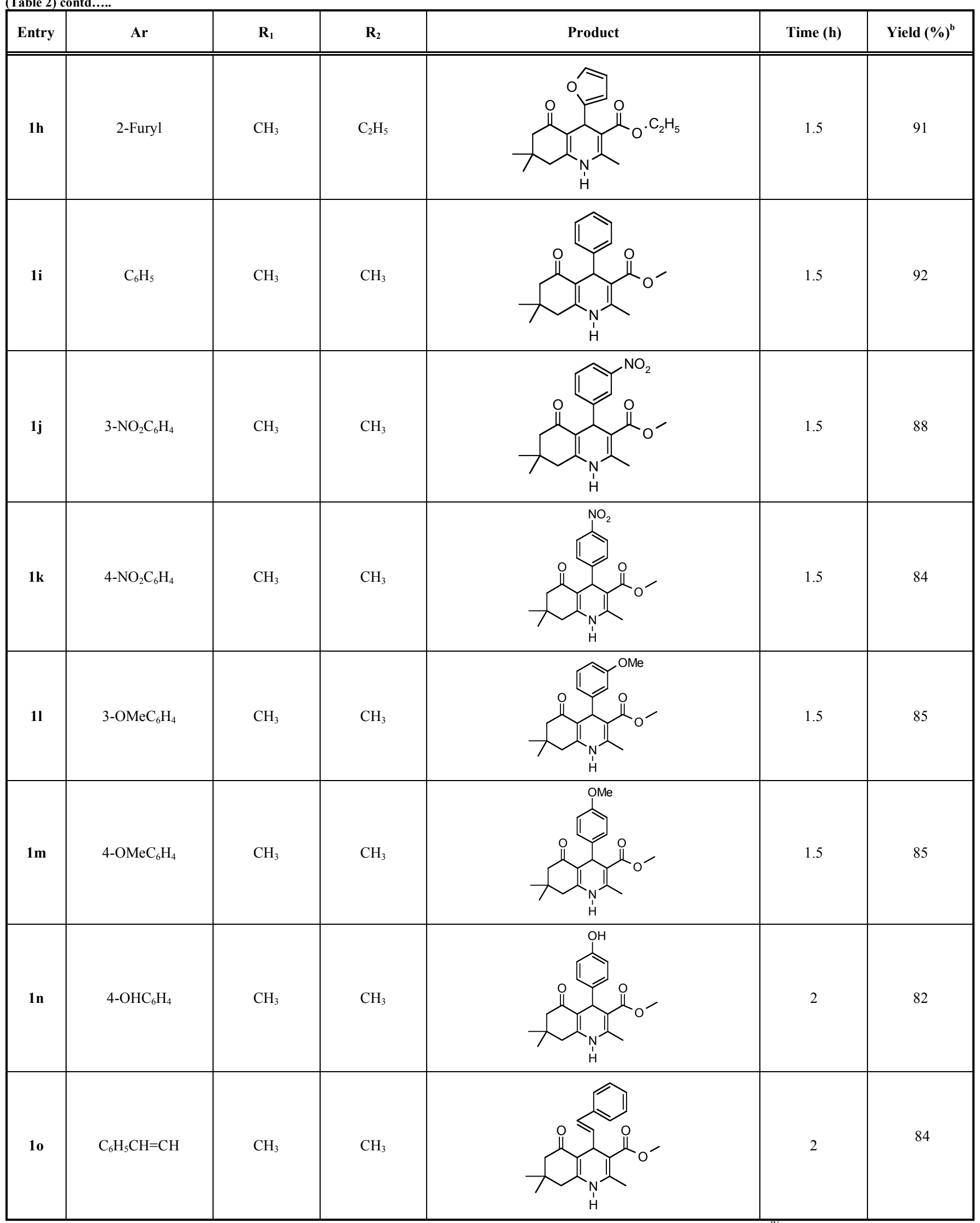

${ }^{\mathrm{a}}$ Reaction condition- aldehydes $(1 \mathrm{mmol})$, ethyl acetoacetate $(1 \mathrm{mmol})$, dimedone $(1 \mathrm{mmol})$, ammonium acetate $(1 \mathrm{mmol})$ and catalyst $\left(\mathrm{NH}_{4}\right)_{6}\left[\mathrm{Mn}^{\mathrm{IV}} \mathrm{Mo}_{9} \mathrm{O}_{32}\right](2 \mathrm{~mol} \%)$ in $2.5 \mathrm{ml}$ ethanol at room temperature. 
${ }^{13} \mathrm{C} \mathrm{NMR}\left(\mathrm{CDCl}_{3}, 75 \mathrm{MHz}\right): \mathrm{d} 14.17,19.48,27.08,29.37$, $32.77,36.96,40.96,50.55,60.07,105.11,111.25,121.30$, $122.84,128.57,134.83,144.41,148.27,149.19,166.91$, 195.47 .

IR (KBr):3292,3191, 3079, 2960, 1639, $1529 \mathrm{~cm}^{-1}$. MS (ESI) $\mathrm{m} / \mathrm{z}(\mathrm{M}+\mathrm{H})^{+}$Calculated for $\mathrm{C}_{21} \mathrm{H}_{25} \mathrm{~N}_{2} \mathrm{O}_{5}: 385$.2. Found: 385 .

\section{2,7,7-Trimethyl-5-Oxo-4-(3-Methoxyphenyl)-1,4,5,6,7,8- Hexahydroquinoline-3-Carboxylic Acid Ethyl Ester (1d)}

${ }^{1} \mathrm{H}$ NMR $\left(\mathrm{CDCl}_{3}, 300 \mathrm{MHz}\right): \mathrm{d} 0.92$ (s, 3H), 1.03 (s, 3H), $1.19(\mathrm{t}, \mathrm{J} 1 / 47.1 \mathrm{~Hz}, 3 \mathrm{H}), 1.96-2.53(\mathrm{~m}, 8 \mathrm{H}), 3.72(\mathrm{~s}, 1 \mathrm{H}), 4.04$ $(\mathrm{q}, \mathrm{J} 1 / 47.1 \mathrm{~Hz}, 2 \mathrm{H}), 4.95(\mathrm{~s}, 1 \mathrm{H}), 6.33(\mathrm{~s}, 1 \mathrm{H}), 6.62(\mathrm{~m}, 1 \mathrm{H})$, $6.85(\mathrm{~m}, 2 \mathrm{H}), 7.09(\mathrm{~m}, 1 \mathrm{H})$

${ }^{13} \mathrm{C}$ NMR $\left(\mathrm{CDCl}_{3}, 75 \mathrm{MHz}\right)$ : d 14.25, 19.36, 27.20, 29.39, 32.68, 36.49, 41, 50.73, 55.06, 59.81, 105.87, 111.03, $114.09,120.59,128.72,148.64,159.26,167.45,195.61$.

IR (KBr):3302, 2958, 1644, 1610, $1484 \mathrm{~cm}^{-1}$. MS (ESI) $\mathrm{m} / \mathrm{z}(\mathrm{M}+\mathrm{H})^{+}$Calculated for $\mathrm{C}_{22} \mathrm{H}_{27} \mathrm{NO}_{4}$ : 369.4 Found: 369 .

\section{ACKNOWLEDGMENT}

A.R. Supale gratefully acknowledges UGC, New Delhi for Research fellowship.

\section{REFERENCES}

[1] (a) Di Stilo, A.; Visentin, S.; Clara, C.; Gasco, A.M.; Ermondi, G.; Gasco, A. New 1,4-Dihydropyridines conjugated to furoxanyl moieties, endowed with both nitric oxide-like and calcium channel antagonist vasodilator activities. J. Med. Chem., 1998, 4l(27), 5393-5401. (b) Sawada, Y.; Kayakiri, H.; Abe, Y.; Mizutani, T.; Inamura, N.; Asano, M.; Hatori, C.; Arsmori, I.; Oku, T.; Tanaka, H. Discovery of the first non-peptide full agonists for the human bradykinin B2 receptor incorporating 4-(2-Picolyloxy)quinoline and 1-(2-Picolyl)benzimidazole frameworks. J. Med. Chem. 2004, 47(11), 2853-2863. (c) Shan, R.; Velazquez, C.; Knaus, E.E. Syntheses, calcium channel Agonist-Antagonist modulation activities, and nitric oxide release studies of Nitrooxyalkyl 1,4-Dihydro-2,6dimethyl-3-nitro-4-(2,1,3-benzoxadiazol-4-yl)pyridine-5-carboxylate racemates, enantiomers, and diastereomers. J. Med. Chem., 2004, 47(1), 254-261. (d) Suarez, M.; Verdecia, Y.; Illescas, B.; MartinezAlvarez, R.; Avarez, A.; Ochoa, E.; Seoane, C.; Kayali, N.; Martin, $\mathrm{N}$. Synthesis and study of novel fulleropyrrolidines bearing biologically active 1,4-dihydropyridines. Tetrahedron, 2003, 59(46), 9179-9186.

[2] (a) Buhler, F.R.; Kiowski, W.J. Calcium antagonists in Hypertension. Hypertension 1987, 5, S3-10. (b) Reid, J.L.; Meredith, P.A.; Pasanisi, F. Clinical pharmacological aspects of calcium antagonists and their therapeutic role in Hypertension. J. Cardiovasc. Pharmacol., 1985, 7, S18-20.

[3] (a) Godfraid, T.; Miller, R.; Wibo, M. Calcium antagonism and calcium entry blockade. Pharmacol. Rev., 1986, 38, 321-416. (b) Sausins, A.; Duburs, G. Synthesis of 1,4-dihydropyridines by cyclocondensation reactions. Heterocycles, 1988, 27(1), 269-289. (c) Mannhold, R.; Jablonka, B.; Voigdt, W.; Schoenafinger, K.; Schravan, K. Calcium- and calmodulin-antagonism of elnadipine derivatives: comparative SAR. Eur. J. Med. Chem., 1992, 27, 229235.

[4] (a) Klusa, V. Cerebrocrast, neuroprotectant, cognition enhancer. Drugs Future, 1995, 20, 135. (b) Boer, R.; Gekeler, V. Chemosensitizers in tumor therapy: new compounds promise better efficacy. Drugs Future, 1995, 20, 499. (c) Bretzel, R.G.; Bollen, C.C.;
Maeser, E.; Federlin, K.F. Nephroprotective effects of nitrendipine in hypertensive type I and type II diabetic patients. Am. J. Kidney Dis., 1993, 21, S53-S64.

[5] (a) Loev, B.; Snader, K.M. The hantzsch reaction. I. oxidative dealkylation of certain dihydropyridines. J. Org. Chem., 1965, 30(6), 1914-1916. (b) Hantzsch, A. Synthese von thiazolen und oxazolen. Ber. Dtsch. Chem. Ges., 1888, 21, 942-946. (c) Hantzsch, A. Neue Bildungsweise von Pyrrolderivaten. Ber. Dtsch. Chem. Ges., 1890, 23, 1474-1476. (d) Dondoni, A.; Massi, A.; Minghini, E.; Bertolasi, V. Multicomponent Hantzsch cyclocondensation as a route to highly functionalized 2- and 4-dihydropyridylalanines, 2and 4-pyridylalanines, and their $\mathrm{N}$-oxides: preparation via a polymer-assisted solution-phase approach. Tetrahedron, 2004, 60(10), 2311-2326.

[6] (a) Agarwal, A.; Chauhan, P.M.S. Solid supported synthesis of structurally diverse dihydropyrido[2,3- $d]$ pyrimidines using microwave irradiation. Tetrahedron Lett., 2005, 46(8), 1345-1348. (b) Ji, S.-J.; Jiang, Z.-Q.; Lu, J.; Loh, T.-P. Facile ionic liquidspromoted one-pot synthesis of polyhydroquinoline derivatives under solvent free conditions. Synlett, 2004, 831-835. (c) Zolfigol, M.A.; Safaiee, M. Synlett, 2004, 827. (d) Moseley, J.D. Alternative esters in the synthesis of ZD0947. Tetrahedron Lett., 2005, 46, 3179-3181. (e) Ko. S.; Yao, C.-F. Ceric Ammonium Nitrate (CAN) catalyzes the one-pot synthesis of polyhydroquinoline via the Hantzsch reaction. Tetrahedron, 2006, 62(31), 7293-7299. and references cited therein. (f) Mekheimer, R.A.; Hameed, A.A.; Sadek, K.U. Solar thermochemical reactions: four-component synthesis of polyhydroquinoline derivatives induced by solar thermal energy. Green Chem., 2008, 10, 592-593. (g) Kumar A.; Maurya R.A. Synthesis of polyhydroquinoline derivatives through unsymmetric Hantzsch reaction using organocatalysts. Tetrahedron, 2007, 63(9), 1946-1952.

[7] (a) Pope, M.T. Isopoly and Heteropoly Anions; Springer: Berlin, Germany, 1983. (b) Muller, A. Polyoxometalate Chemistry; Kluwer Academic: Dordrecht, The Netherlands, 2001. (c) Kozhevnikov, I.V. Catalysis by Polyoxometalates; Wiley: Chichester, UK, 2002. (d) Kozhevnikov, I.V. Catalysis by heteropoly acids and multicomponent polyoxometalates in liquid-phase reactions. Chem. Rev., 1998, 98(1), 171-198.

[8] (a) Bhosale, B.D.; Gokavi, G.S. Kinetics and mechanism of oxidation of arsenous acid by Waugh-type enneamolybdomanganate. Indian J. Chem. A, 2006, 45, 398-401. (b) Gurame, V.M.; Gokavi, G.S. Kinetics and mechanism of oxidation of hypophosphite by Waugh-type enneamolybdomanganate(IV) in Perchloric acid. Polyhedron, 2008, 27, 1905-1910.

[9] (a) Supale, A.R.; Gokavi, G.S. Chromium (III) catalyzed selective oxidation of sulfides to sulfoxides using $30 \% \mathrm{H}_{2} \mathrm{O}_{2}$. Catal. Lett., 2008, 124, 284-287. (b) Supale, A.R.; Gokavi, G.S. Oxidation of thiols to disulfides using $\mathrm{H}_{2} \mathrm{O}_{2}$ catalyzed by recyclable chromic potassium sulphate at room temperature. React. Kinet. Catal. Lett. 2008, 93(1), 141-148. (c) Mardur, S.P. Gokavi, G.S. Heteropoly acid catalyzed synthesis of 3,4-dihydropyrimidin-2(1H)-ones. Catal. Commun., 2007, 8, 279-284. (d) Mardur, S.P.; Halligudi, S.B.; Gokavi, G.S. Oxidation of aliphatic and benzylic alcohols by Oxone $^{\mathrm{R}}$, catalysed by 12-tungstocobaltate (II). Catal. Lett., 2004, 96, 165-167.

[10] Baker, L.C.W.; Weakly, T.J.R. The stabilities of the 9-molybdomanganate (IV) and 9-molybdonickelate (IV) ions. J. Inorg. Nucl. Chem., 1966, 28(2), 447-454.

[11] (a) Nakamoto, K. Infrared and Raman spectra of Inorganic Compounds, $3^{\text {rd }}$ ed.; John Wiley \& Sons: New York, 1977, p. 115. (b) Martin, C.; Lamonier, C.; Fournier, M.; Mentre, O.; Harle, V.; Guillaume, D.; Payen, E. Preparation and characterization of 6molybdocobaltate and 6 -molybdoaluminate cobalt salts: evidence of a new heteropolymolybdate structure. Inorg. Chem., 2004, 43, 4636-44. (c) Tsuboi, M. Vibrational spectra of phosphite and hypophosphite anions, and the characteristic frequencies of $\mathrm{PO}_{3}^{--}$and $\mathrm{PO}_{2}{ }^{-}$groups. J. Am. Chem. Soc., 1957, 79(6), 1351-1354. 